\title{
Adaptation of Barn Owl Localization System with Spike Timing Dependent Plasticity
}

\author{
Juan Huo, Alan Murray, Leslie Smith, Zhijun Yang
}

\begin{abstract}
To localize a seen object, the superior colliculus of the barn owl integrates the visual and auditory localization cues which are accessed from the sensory system of the brain. These cues are formed as visual and auditory maps, thus the alignment between visual and auditory maps is very important for accurate localization in prey behavior. Blindness or prism wearing may disturb this alignment. The juvenile barn owl could adapt its auditory map to this mismatch after several weeks training. Here we investigate this process by building a computational model of auditory and visual integration with map adjustment in the deep superior colliculus. The adaptation is based on activity dependent axon developing which is instructed by an inhibitory network. In the inhibitory network, the strength of the inhibition is adjusted by spike timing dependent plasticity(STDP). The simulation results are in line with the biological experiment and support the idea that the STDP is involved in the alignment of sensory maps. The system of the model provides a new mechanism capable of eliminating the disparity in visual and auditory map integration.
\end{abstract}

\section{INTRODUCTION}

Vision and hearing are the most important senses for many mammals and birds and both visual and auditory cues are used to locate an object of interest. Although the cognitive pathways and the organization of sensor organs are disparate, we experience the world as single place. When a disparity occurs, or is introduced, between different sensory pathways, objects cannot be localized correctly. One wellstudied example is that of the realignment between visual and auditory maps of space in the superior colliculus (SC) of the barn owl [1], [2]. Furthermore, it has been shown that a lesion in the visual map can cause the auditory map to degrade [3]. To explore the interaction between visual and auditory maps in a more controlled manner, experiments have been conducted, in which a juvenile barn owl wears a prism over its eyes, shifting the visual map laterally [2]. Unsurprisingly, it loses precision in hunting. However, readjustment of the auditory map to match the visual map after weeks of training allows the barn owl to recapture its hunting ability. This indicates that the link between auditory and visual maps is plastic and that it can realign on the basis of trial-and-error experience [4]. This paper presents a model to explore and to understand the biological mechanism with activity dependent axon developing modulated by spike timing dependent plasticity(STDP). STDP is a learning rule

Juan Huo, Alan Murray and Zhijun Yang are with the Electronics \& Engineering School, Edinburgh University, Edinburgh, UK (phone: 01316505664; fax:01316506554; email: \{J.Huo, Zhijun.Yang, Alan.Murray\}@ed.ac.uk).

Leslie Smith is with the department of computing science and mathematics, University of Stirling, Stirling, UK (phone:(01786)467435; fax:(01786)464551; email:1ss@cs.stir.ac.uk). in which the synapse weight is strengthened or weakened by the paired presynaptic spikes and postsynaptic spikes in a time window [5]. The model captures many of the features of the biological system and replicates many results from biological experiments.

\section{BIOLOGICAL BACKGROUND}

In the barn owl, visual and auditory pathways are believed to be integrated in the deeper layer of SC, which is a paired structure that is part of the brain's tectal area [6]. The deeper layers of SC are also connected to many sensorimotor areas of brain and the SC is involved in orientation initiated behavior such as eye saccades. In the superficial layer of SC, visual and auditory maps of spatial localization are accessed from the other parts of the brain separately [2]. Most of the neurons in the deep SC are bimodal neurons which can react to both visual and auditory stimuli.

Visual stimuli are elicited from the retina and projected to the superficial SC in such a way that a particular SC neuron will respond to visual input from a particular location in the retinal map [7].

The auditory stimuli to the SC comes from external nucleus of the inferior colliculus (ICx), Figure 1 [8], [1]. The remainder of inferior colliculus (IC), the central nucleus (ICc) which is connected to previous auditory system , occupies most of IC area and the ICx wraps around it. The auditory information conveyed from ICc to ICx experiences a converging process. The topography of neural activity in both ICc and ICx is sensitive to the interaural time difference (ITD) which is the auditory localization cue of azimuth [9]. The auditory space map is created by integrate ITD information across the frequency channels. This converging happens in the projection from the tonotopically organized ICc to ICx. The neurons of ICc within the same ITD laminae but different frequency response are connected to ICx neuron via many points to one point projection.

The auditory maps formed in the ICx and the ICc are also different for their sensitivity to changes in the visual map. The projection of auditory information on to the ICx is able to adapt as the visual map change in SC is shifted (for example, by a prism), whereas ICc neurons do not react to such a change [2]. Axon sprouting and/or retraction (axonogenesis) and synapse formation/removal (synatogenesis) in the ICx give rise to this auditory map adaptation [10], [8], while ICc neurons are largely initial receptors of auditory information in IC.

The auditory map adaptation is instructed by the visual response which has been detected in ICx when GABAergic 
inhibition is blocked in SC [11]. It is believed the SC provides a visual space template topographically representing the visual space for the ICx map shift. The visual instructive signal is modulated by an inhibitory network in SC. The instructive visual activity does not excite ICx neuron if visual and auditory localization cues are consistent, but it is strong if visual and auditory maps are not in register.

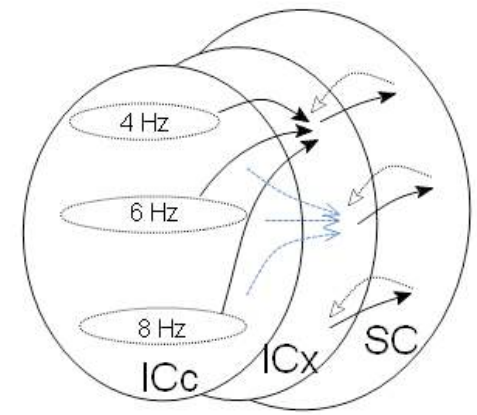

Fig. 1. The structure of ICc and ICx. The IC is composed of ICc and ICx, ICx wraps around ICc and takes less area than ICc. The arrows are the projections between different layers. The hollow arrows send the visual instructive signals from SC neurons to ICx neurons. The arrows with thin head are the new connections resulting from prism experience. This figure is modified from [8], [12].

The superior colliculus of the barn owl has been studied extensively [1], [13], [14]. Among them, [1] shows the hebbian learning mechanism in establishing and maintaining the map alignment. However, the neurons and network connections in [1] are non physiological. [14] explained the visual and auditory input integration in the bimodal neuron with a nonlinear neuron model, but no adaptation and plasticity are noted. [13] has a good network structure of STDP. Although the structural plasticity is well explained, the axonogenesis hasn't been referred to in [13], whereas [12] indicates that the prism experience must induce axonal sprouting and synapstogenesis.

With the recent new physiological data from biological experiments [15], [16], [11], [10], [12], our model introduces the axon growth factor (neurotrophin) for the modeling of axonogenesis between ICc and ICx in barn owl SC. The axonal projection between ICc and ICx is directed by neurotrophin of the target ICx neurons. For better explaining the instructive learning, visual instructive signal is shown to be generated in an inhibitory network in SC. The strength of the inhibition in this network is modulated by the STDP which modified the weight of the sensory input synapses connected to the bimodal neurons of SC [5].

The results of this model are consistent with the biological experiment. The ICx map shift in accord with the visual space changed by prism. This model provides a better understanding of the mechanism in map alignment and support the idea that STDP is involved in the adaptation for spatial localization.

\section{Methods}

As shown in Fig. 2, we set the virtual owl positioned at the center of a fixed, head centered reference system with the origin centered on the perch, the target moves along the semicircular track. This model is based on neural spike computation. The target position in the visual and auditory maps are translated into input spike trains generated in section III-A. The model in the simulation consists of 10 pathways as described in section III-B, each pathway corresponding to an azimuth localization cue around $12^{\circ}$.

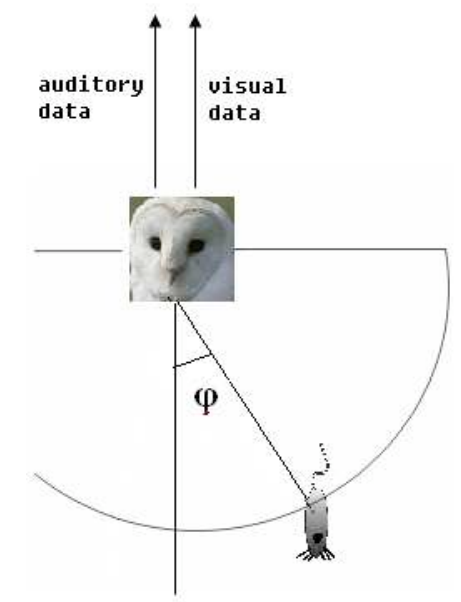

Fig. 2. Overall virtual simulation environment. The position of the target depends on the owl head.

\section{A. Input Spike Train Generating}

We assume the visual stimuli are generated by luminous points which are projected to retina. According to the onoff activation of the ganglion cells in retina, the neurons corresponding to the target localization cue have the strongest response. Similarly, the most active neurons of auditory map in ICx is the one with the character ITD of the target localization in space. Therefore, the visual and auditory inputs with the same localization cue are correlated with each other.

The spike train group is produced in an arbitrary way with manually generated spike templates but is more possible to be applied in artificial systems, for example robot. The spike sequence and the time interval between spikes in the spike template is set manually. Each template lasts 50 time units while their firing period lasts 20 time units. The firing period of these templates can be classified as strong template with high intensity and weak template with low intensity. The visual strong template matches the auditory strong template and they increase the synaptic weight when they are put together. But the strong template does not match the weak template and they decrease the synaptic weight by STDP. The whole spike train is generated by replicating the template. By 
this way, the amount of synaptic weight change and the time of the change are controlled more accurately.

\section{B. The Network Structure}

The whole network with 10 pathways is shown in Fig.4. The schematic of a single visual and auditory signal processing pathway is shown in Fig.3. The single pathway can be divided into two basic blocks. The block I includes an instructive learning between ICc and ICx layer. The axonal projection between ICc and ICx is guided by visual response which comes from block II (the inhibitory neural network), while the output of ICx layer is the auditory input of block II.

The axonogenesis and synaptogenesis between ICx and ICc is affected by the guidance factor [17], [18]. The activity of the visual response from block II in the ICx triggered the release of the guidance factor of axon called neurotrophin. The dynamic axon extension with the motile sensory structure, axon growth cone, from the projection source ICc, keeps seeking its synaptic target via the guidance factor.

$$
n\left(y_{c e n}\right)=\sum_{y_{I C x}} B\left(y_{I C x}\right) D\left(y_{I C x}-y_{c e n}\right)
$$

In the model, the neurotrophin $\mathrm{n}(\mathrm{y})$ presents at the yth neuron of ICx layer. The excitation of every ICx neuron at node $y_{I C x}$ contributes to the neurotrophin accumulation, but the degree of their contribution varied depends on their distance with the activity center $y_{c e n} . B_{I C x}$ is proportional to postsynaptic activity of ICx neuron $y_{I C x}$ and weighted by a spreading kernel $D\left(y_{I C x}-y_{c e n}\right)$ [19]. $D\left(y_{I C x}-y_{c e n}\right)$ is exponentially decayed with distance $\left\|y_{I C x}-y_{c e n}\right\|$ from the neurotrophin release center $y_{c e n}$.

For the axon sprouting layer ICc, the growth cone activity is bounded by the presyanptic factor which is a summation filter representing the linear sum of the presynaptic spikes of the corresponding neuron. The most active growth cone has the highest possibility to be extended.

The direction of the developing growth cone is computed by identifying the node $y_{t a g} \subset Y\left(y_{t a g}\right), Y\left(y_{t a g}\right)$ is the assemble of ICx neurons. $y_{t a g}$ is the right direction of growth cone with the most neurotrophin. As the new axonal connection is growing, we assume that axon conduction block occurs at points of bifurcation along the entire length of the presynaptic nerve and inhibits the old connection [20].

$$
y_{\text {tag }}=\operatorname{argmax}_{x \in Y\left(y_{\text {tag }}\right)} n(x)
$$

The block II of this model is the inhibitory neural network with the STDP based integration of visual and auditory signal on SC neuron. The source of the visual instructive signal in the ICx layer comes from the SC inter neuron. The strength of the visual response is controlled by the inhibitory network in the SC. The inhibitory neural network is composed by an $\mathrm{SC}$ bimodal neuron and an inter neuron. The bimodal neuron can response to either visual input or auditory input. In order to access these inputs, it is connected with two excitatory synapses whose strength is adjusted by STDP. The bimodal

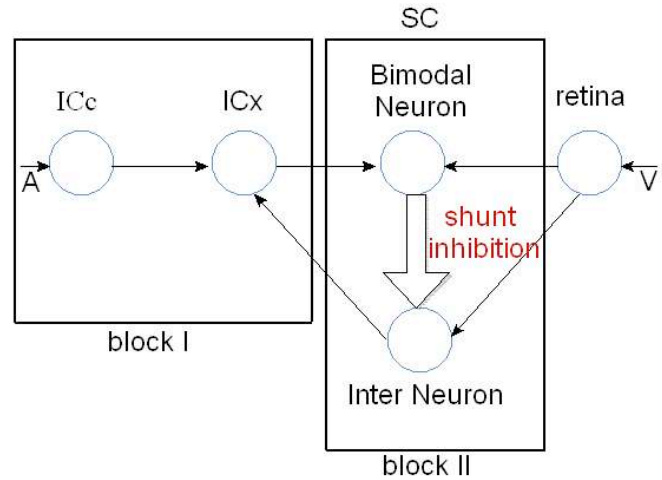

Fig. 3. The schematic of auditory and visual signal processing pathway. Neurons are labeled and shown as circles, excitatory connections are depicted as filled thin arrows, the inhibitory connection between SC bimodal neuron and SC inter neuron is an open arrow. Auditory input (A) represents the peak auditory response, namely the target site in the auditory map. Visual input (V) indicates the target site in the visual map. If $\mathrm{A}$ and $\mathrm{V}$ arrive at the same pathway, this indicates SC bimodal neuron gets two strong correlated input spike trains. The synapses connected to the bimodal neuron will be strengthen, the output spike number is highly increased and so is the inhibition for the inter neuron. In contrast, the auditory and visual disparity weakens the synapses and reduces the inhibition.

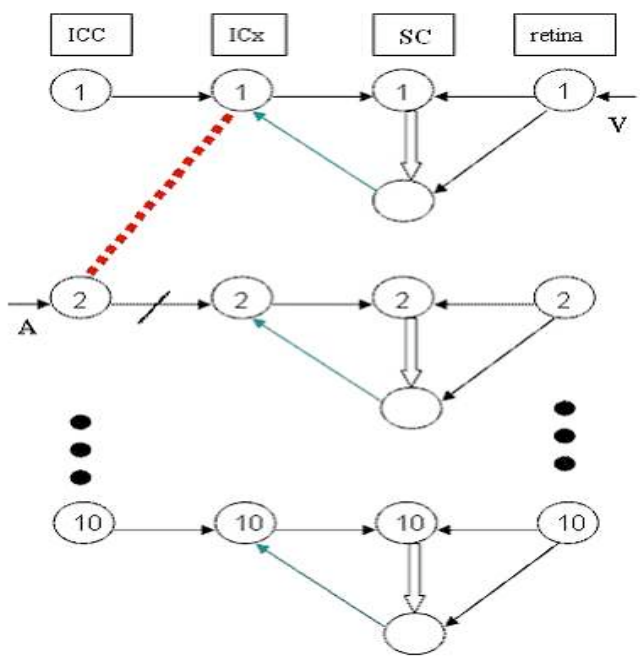

Fig. 4. Schematic of the network after visual and auditory re-alignment after the owl wearing the prism. Since the visual stimuli arrives at retina-1, ICx-1 receives the strongest visual guiding signal. But the auditory stimuli arrives at $\mathrm{ICc}-2$, the axon growth cone from ICc-2 is attracted by the neutrophin released by ICx-1 and the new connection is built, which is depicted as dashed line between ICc- 2 and ICx-1. Although the original connection between ICc- 2 and ICx-2 is kept, the information path is blocked due to axonal conductance depression. 
neuron is also connected with an inhibitory synapse between it and the inter neuron. Inter neuron is also the SC neuron but it is not clear until now whether it is bimodal neuron or not [11]. Hereby, we set the inter neuron as a neuron independent of the bimodal neuron.

The output spikes of SC neuron are the inputs of the inter neuron through the inhibitory synapses. Therefore, the output spike number of inter neuron is deducted and the deduction is proportional to the spikes number of SC neuron output.

\section{The Neuron Model}

The neuron we used in the model is leaky integrate and fire neuron with both excitatory synapses and inhibitory synapses. Here $g_{e}$ is the excitatory synaptic conductance, it is together with excitatory reversal potential $V_{\text {exc }} . g_{i}$ is the inhibitory conductance, it is accompanied with inhibitory reversal potential $V_{i n h}$.

$C \frac{d V(t)}{d t}=-g_{l}\left(V(t)-V_{\text {rest }}\right)-g_{e}\left(V(t)-V_{e x c}\right)-g_{i}\left(V(t)-V_{\text {inh }}\right)$

Usually the excitatory reversal potential $V_{\text {exc }}$ is around $0 \mathrm{mV}$. The inhibitory reversal potential $V_{i n h}$ is about the resting potential. This is called shunting inhibition [7] which decreases the firing rate by increasing the inhibition. Parameters: $V_{\text {rest }}=-70 \mathrm{mV}, V_{\text {exc }}=0 \mathrm{mV}, V_{\text {inh }}=-70 \mathrm{mV}$, $\tau_{m}=5 \mathrm{~ms}$.

\section{RESUlTS}

In the simulation, we assume the GABAergic inhibition of visual instructive signal has been blocked [16]. Here we analyze the details of the system performance.

\section{A. Axon Updates in the Block I}

The initial state of this system is a normal barn owl, the visual and auditory map are registered. After prism learning, the auditory map in ICx shifts. During the prism learning process, each time the sensory stimuli come, neurotrophin is released by postsyantpically active ICx neurons shown in Fig.5. When the maximal neurotrophic threshold is reached, the neurotrophin is reset to the original state. The ICc node with the most active growth cone swaps its target location with the ICx node where the neurotrophic threshold is reached. New axonal connection is established while the old connection is inhibited.

The axon arrangement initially is in table I, the ICc neuron is numbered as source neuron and projected to corresponding ICx neuron by axons. After the prism learning process with prism angle $35^{\circ}$, auditory map in ICx was moved forward in terms of the prism angle. At the same time, the old connection is blocked. The axonal arrangement is changed as shown in table II.

\section{B. The Plasticity in the Inhibitory Neural Network}

The map shift is introduced by the visual stimuli. In the normal barn owls, the visual response dose not appear in the ICx as long as sound localization cues are represented

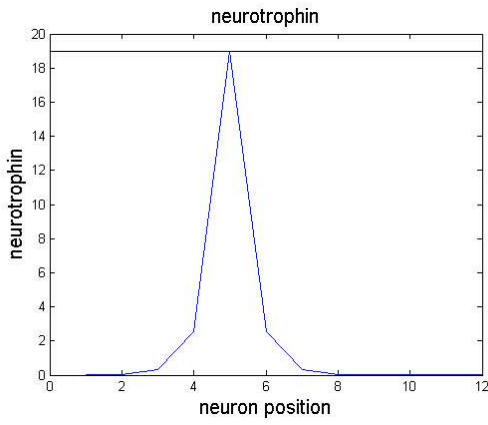

Fig. 5. Plot of neutrophin contributed by the target ICx neurons. Here the activity of the 5th ICx neuron is strong enough to be identified as the maximum neurotropic update.

TABLE I

BEFORE PRISM LEARNING

\begin{tabular}{|l|llllllllll|}
\hline source & 1 & 2 & 3 & 4 & 5 & 6 & 7 & 8 & 9 & 10 \\
target & 1 & 2 & 3 & 4 & 5 & 6 & 7 & 8 & 9 & 10 \\
\hline
\end{tabular}

correctly in the space map. When the A-V mismatch is introduced by prism, the visual response is strong in the ICx [11]. The inhibitory neural network in the block II is used to encode this mismatch.

Here we test pathway 1 in Fig.4. This test starts from the prism wearing, we presume the barn owl gets rid of its prism from the 10th time bin and then wears it again. The purpose of this arrangement is to prove the possibility of the original connection recovery. In natural way, when the barn owl gets rid of its prism, it can recover its original connections between visual and auditory map. Learning process for map adjustment will take weeks. To simplify this procedure with continuous excitation, the time scale is drasticly reduced. The visual input stimuli to the learning pathway is strong with high firing spike rate. Because of the visual and auditory map disparity, the auditory stimuli are weak at first, auditory spike train and visual spike train are uncorrelated with each other. Low correlation decreased the synaptic weight of the two excitatory synapses connected to SC and reduced the spike output number of SC in terms of STDP [21]. The weakened inhibition of the inter neuron increases its spike output, namely the visual activity in the ICx. This leads to the release of neurotrophin and ICc axon growth cone uptake. When the new connection is built for this pathway, high firing rate auditory spike train replaces the low firing rate spike train. Since the visual and auditory signal come from the same target source, with STDP, the increased correlation between visual and auditory inputs strengthen the synaptic

TABLE II

AFTER PRISM LEARNING

\begin{tabular}{|l|cccccccccc|}
\hline source & 1 & 2 & 3 & 4 & 5 & 6 & 7 & 8 & 9 & 10 \\
target & 3 & 4 & 5 & 6 & 7 & 8 & 9 & 10 & - & - \\
\hline
\end{tabular}


weight and trigger more SC spike outputs. This increases the inhibition towards the inter neuron, as shown in Fig. 6(b) during the 6th and 10th time bin, not any inter neuron spike is generated. It means visual and auditory maps are coupled again in this period.

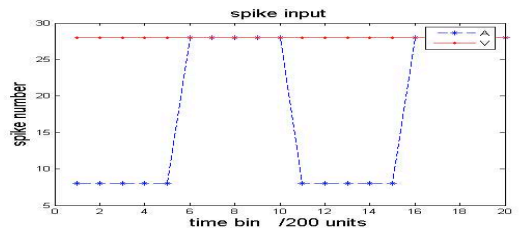

(a)

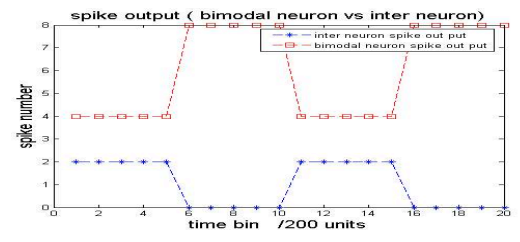

(b)

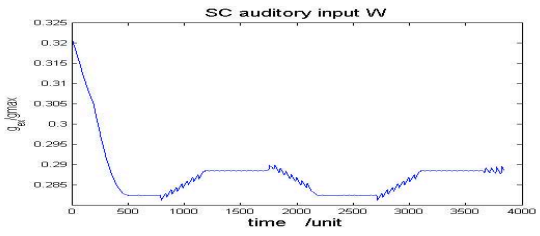

(c)

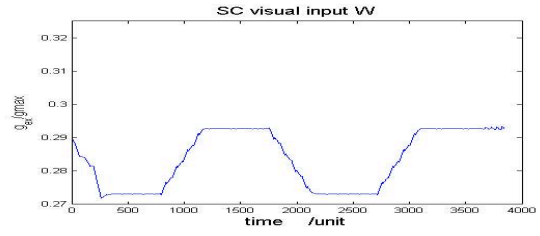

(d)

Fig. 6. The statistics of the input and output spikes in the inhibitory network.(a) The input spike pattern to the SC bimodal neuron. The visual stimuli is strong while the auditory spike train follows the change of the connection between ICc and ICx. (b) The output spike pattern comparison between SC bimodal neuron and interneuron. The target auditory localization is $\mathrm{nSC}$ in this pathway because of the A-V disparity during the first 5 time bins. When the ICc axon growth cone is switched to this pathway, the auditory stimuli become strong and the spike firing rate becomes high again. This increases the correlation between visual and auditory input spike train. The strengthened inhibition of inter neuron decreased the activity of the visual response. (c)(d) are the synaptic weight change of the two excitatory synapses for the inhibitory neural network plasticity test. (c) the synapse from ICx neuron to SC. (d) the synapse from retina to SC.

\section{CONCLUSION}

In this paper, we demonstrated a model with the essential properties of SC sensory integration with the newest biological discoveries. This is a deeper view in plastic alignment between sensory maps in barn owl superior colliculus. It provides a novel structure to explain the mechanism of the visual and auditory signal integration, which is more approaching the real biosystem than previous work. STDP together with inhibitory network controlled the visual instructive signal of ICx which induces the axogenesis and synaptogenesis between ICc and ICx neurons. Plasticity of this mechanism eliminates the visual and auditory disparity for the target localization. On the other hand, this model may provide a new solution for engineering design of sensor fusion system or robotic system. In the real environment, adaptivity is a crucial issue in the design of autonomous system, which is the advantage of biological individuals comparing to artificial intelligent system.

Since axon growing and new synapse formation are simplified in this model, further investigation will shed more light on the different activity between new and old axon terminal, because it's not clear until now how the old connection existing without activity. The inhibition of the original connection is assumed to be the axon bifurcation block. However, the axon terminal synaptic depression may be another method and could possibly explain why the barn owl reactivate its visuomotor behavior in a very short time after the prism removing. Although at present the model is simulated under the above virtual environment, a database of real-world sensory stimuli in later study could help a real-time application and possible robotic implementation in future work.

\section{ACKNOWLEDGEMENT}

We would like to thank EPSRC's financial support, Sim Bamford for his suggestion which triggered our initial interesting in saccadic system. We would also thank Leslie Smith for his support in the STDP Matlab coding.

\section{APPENDIX}

STDP rules is consistent with the basic Hebbian learning mechanism. It adapts synaptic weights by pairing pre- and postsynaptic action potentials within a time window (Figure 7). A weight is increased when a pre-synaptic spike precedes a post-synaptic spike and the weight is decreased when the post-synaptic spike arrives first [21]. According to [5], the STDP in this paper is set as follows. The parameters $\tau_{+}=$ $\tau_{-}=20 \mathrm{~ms}$ determine the ranges of pre-to-postsynaptic inter spike intervals over which synaptic strengthening and weakening occur, $W_{-}=1.05 W_{+}=0.003$ determine the maximum amounts of synaptic modification.

$$
\Delta W=\left\{\begin{aligned}
W_{+} \exp \left(\Delta t / \tau_{+}\right) & \text {if } \Delta t<0 \\
-W_{-} \exp \left(\Delta t / \tau_{-}\right) & \text {if } \Delta t \geq 0
\end{aligned}\right.
$$

\section{REFERENCES}

[1] M. Rucci, G. Tononi, and G. M. Edelman, "Registration of neural maps through value-dependent learning: modeling the alignment of auditory and visual maps in the barn owl's optic tectum," J Neurosci., 1997.

[2] E. Knudsen, "Auditory and visual maps of space in the optic tectum of the owl," The Journal of Neuroscience, 1982.

[3] K. EI, "Early blindness results in a degraded auditory map of space in the optic tectum of the barn owl," Proc Natl Acad Sci, vol. 85, no. 16, pp. 6211-4, 1988.

[4] E. Knudsen and P. Knudsen, "Visuomotor adaptation to displacing prisms by adult and baby barn owls," Journal of Neuroscience, vol. 9, pp. 3297-3305, 1989. 


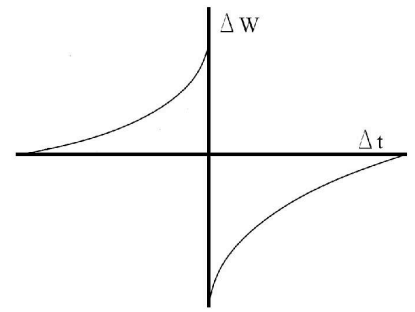

Fig. 7. Learning Window for STDP. $\Delta t=t_{\text {pre }}-t_{\text {post }}, \Delta \mathrm{W}$ refers to the amount of weight change.

[5] S. Song, K. D. Miller, and L.F.Abbott, "Competitive hebbian learning through spike-timing-dependent synaptic plasticity," Nature Neurosci, vol. 3, pp. 919-926, 2000

[6] W. C.Hall and A. Moschovakis, the superior colliculus: new approaches for studying sensorimotor integration. CRC Press, 2004.

[7] L.F.Abbott and P. Dayan, Theoretical Neuroscience. Cambridge: MIT Press, August 2001

[8] J. I.Gold and K. EI, "Adaptive adjustment of connectivity in the inferior colliculus revealed by focal pharmacological inactivation," $J$ Neurophysiol., vol. 85(4), pp. 1575-84, 2001.

[9] T. T. Takahashi, A. D. S. Bala, M. W. Spitzer, D. R. Euston, M. L.Spezio, and C. H. Keller, "The synthesis and use of the owl's auditory space map,” Biol. Cybern., vol. 89, pp. 378-387, 2003.

[10] H. PS and K. EI, "Topographic projection from the optic tectum to the auditory space map in the inferior colliculus of the barn owl," $J$ Comp Neurol, vol. 421, pp. 146-160, 2000.

[11] Y. Gutfreund, W. Zheng, and E. I. Knudsen, "Gated visual input to the central auditory system,” Science, vol. 5586 (297), pp. 1556-9, 2002.

[12] E. I. Knudsen, "Instructed learning in the auditory localization pathway of the barn owl," Nature, vol. 417, no. 6886, pp. 322-328, May 2002.

[13] S. P.Mysore and S. R. Quartz, "Modeling structural plasticity in the barn owl auditory localization system with a spike-time dependent hebbian learning rule," in Neural Networks, 2005. IJCNN '05, vol. 5. 2005 IEEE International Joint Conference, 31 July-4 Aug. 2005, pp. $2766-2771$

[14] Schauer, C. Gross, and H.-M., "Design and optimization of amari neural fields for early auditory-visual integration," in Neural Networks, 2004. Proceedings. 2004 IEEE International Joint Conference, vol. 4. IEEE, July 2004, pp. 2523- 2528.

[15] E. I. Knudsen, W. Zheng, and W. M. DeBello, "Traces of learning in the auditory localization pathway," Proc Natl Acad Sci U S A, vol. 97, no. 22, pp. 11 815-11820, Oct 2000.

[16] Y. Gutfreund and E. I. Knudsen, "Adaptation in the auditory space map of the barn owl," J Neurophysiol., vol. 96, pp. 813-825, 2006.

[17] L. N. Gillespie, "Regulation of axonal growth and guidance by the neurotrophic family of neurotrophic factors," Clinical and Experimental Pharmacology and Physiology, vol. 30, pp. 724-733, 2003.

[18] N. Toni, E. M. Teng, E. A. Bushong, J. B. Aimone, C. Zhao, A. Consiglio, H. van Praag, M. E. Martone, M. H. Ellisman, and F. H Gage, "Synapse formation on neurons born in the adult hippocampus," Nature Neuroscience, vol. 10, pp. 727 - 734, 2007.

[19] B. Taba and K. Boahen, "Silicon growth cones map silicon retina," Advances in Neural Information Processing System, vol. 17, 2006.

[20] H. Hatt and D. O. Smith, "Synaptic depression related to presynaptic axon conduction block," J. Physiol., vol. 259, no. 2, pp. 367-93, 1976.

[21] J. Huo and A. Murray, "The role of membrane threshold and rate in stdp silicon neuron circuit simulation," in Artificial Neural Networks: Formal Models and Their Applications - ICANN, 2005. 